\title{
How Behavior Shapes the Brain and the Brain Shapes Behavior: Insights from Memory Development
}

\author{
Fengji Geng, ${ }^{1,3}$ Morgan Botdorf, ${ }^{2}$ and Tracy Riggins ${ }^{2}$ \\ ${ }^{1}$ Department of Curriculum and Learning Sciences, Zhejiang University, Zijingang Campus, Hangzhou, 310058, ${ }^{2}$ Department of Psychology, \\ University of Maryland, College Park, Maryland 20742, and ${ }^{3}$ Children's Hospital, Zhejiang University School of Medicine, National Clinical \\ Research Center for Child Health, Hangzhou, 310052
}

Source memory improves substantially during childhood. This improvement is thought to be closely related to hippocampal maturation. As previous studies have mainly used cross-sectional designs to assess relations between source memory and hippocampal function, it remains unknown whether changes in the brain precede improvements in memory or vice versa. To address this gap, the current study used an accelerated longitudinal design $(n=200,100$ males) to follow 4- and 6-year-old human children for 3 years. We traced developmental changes in source memory and intrinsic hippocampal functional connectivity and assessed differences between the 4- and 6-year-old cohorts in the predictive relations between source memory changes and intrinsic hippocampal functional connectivity in the absence of a demanding task. Consistent with previous studies, there were age-related increases in source memory and intrinsic functional connectivity between the hippocampus and cortical regions known to be involved during memory encoding. Novel findings showed that changes in memory ability early in life predicted later connectivity between the hippocampus and cortical regions and that intrinsic hippocampal functional connectivity predicted later changes in source memory. These findings suggest that behavioral experience and brain development are interactive, bidirectional processes, such that experience shapes future changes in the brain and the brain shapes future changes in behavior. Results also suggest that both timing and location matter, as the observed effects depended on both children's age and the specific brain ROIs. Together, these findings add critical insight into the interactive relations between cognitive processes and their underlying neurologic bases during development.

Key words: accelerated longitudinal design; brain development; episodic memory; hippocampal functional connectivity; memory development; source memory

\section{Significance Statement}

Cross-sectional studies have shown that the ability to remember the contextual details of previous experiences (i.e., source memory) is related to hippocampal development in childhood. It is unknown whether hippocampal functional changes precede improvements in memory or vice versa. By using an accelerated longitudinal design, we found that early source memory changes predicted later intrinsic hippocampal functional connectivity and that this connectivity predicted later source memory changes. These findings suggest that behavioral experience and brain development are interactive, bidirectional processes, such that experience shapes future changes in the brain and the brain shapes future behavioral changes. Moreover, these interactions varied as a function of children's age and brain region, highlighting the importance of a developmental perspective when investigating brain-behavior interactions.

Received Nov. 6, 2019; revised 0ct. 5, 2020; accepted 0ct. 9, 2020.

Author contributions: F.G., M.B., and T.R. designed research; F.G., M.B., and T.R. performed research; F.G., M.B., and T.R. analyzed data; F.G. wrote the first draft of the paper; F.G., M.B., and T.R. edited the paper; F.G., M.B., and T.R. wrote the paper.

This work was supported by National Institute of Child Health and Human Development Grant HD079518 and the University of Maryland. We thank members of the Neurocognitive Development Lab, especially Kelsey Canada, Elizabeth Mulligan, Marissa Clark, Lisa Cox, Shane Wise, and Jennifer Sloane, for helping with data collection and/or analysis.

The authors declare no competing financial interests.

Correspondence should be addressed to Tracy Riggins at riggins@umd.edu.

https://doi.org/10.1523/JNEUROSCI.2611-19.2020

Copyright $\odot 2021$ the authors

\section{Introduction}

Source memory improves substantially during childhood (e.g., Riggins, 2014). Specifically, with age, children become better at reporting and retaining contextual details of life experiences (Bauer, 2007). This development is closely related to hippocampal maturation, as evidenced by age- and memory-related differences in hippocampal structure and function across development (see Ghetti and Bunge, 2012 for review; Sastre et al., 2016; Tang et al., 2018; Riggins et al., 2020). Previous studies have mainly used cross-sectional designs to assess relations between brain and memory development, which do not allow for investigating true 


\begin{tabular}{lccc}
\multirow{2}{*}{$\begin{array}{l}\text { a) Age-related change } \\
\text { in concurrent relations }\end{array}$} & $\begin{array}{c}\text { Source } \\
\text { memory }\end{array}$ & $\begin{array}{c}\text { Source } \\
\text { memory }\end{array}$ & $\begin{array}{c}\text { Source } \\
\text { memory }\end{array}$ \\
\cline { 2 - 4 } $\begin{array}{l}\text { Wetween behavior and } \\
\text { brain }\end{array}$ & $\begin{array}{c}\text { Functional } \\
\text { connectivity }\end{array}$ & $\begin{array}{c}\text { Functional 2 } \\
\text { connectivity }\end{array}$ & Wave 3 \\
\cline { 2 - 4 }
\end{tabular}
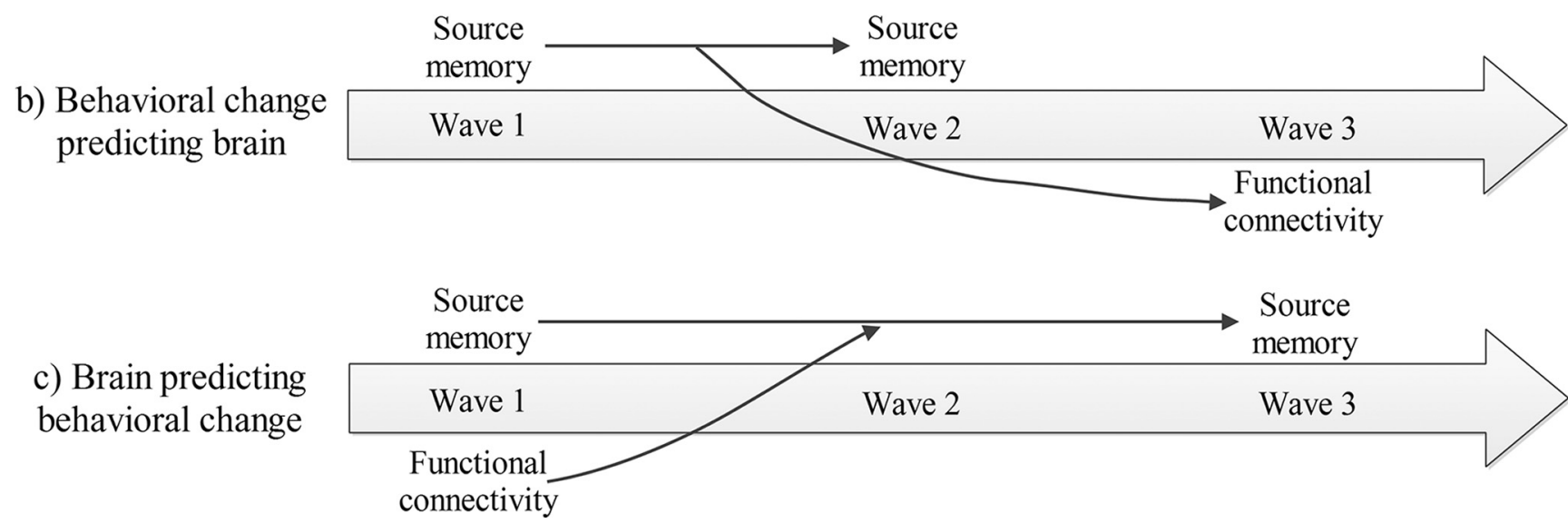

Figure 1. Overview of analyses examining concurrent and predictive relations between source memory and iHFC across development.

developmental changes and may be influenced by confounding factors, such as cohort effects. Therefore, it remains unknown whether changes in the brain precede improvements in memory or vice versa. Additionally, previous studies have focused on either young children or school-aged children, which make comparisons of different developmental periods difficult. To address these gaps, the current study used an accelerated longitudinal design to follow 4- and 6-year-old children for 3 years. This design allowed us to explore developmental changes in intrinsic hippocampal functional connectivity (iHFC) and to assess differences between the 4and 6-year-old cohorts in the predictive relations between source memory and functional connectivity (see Fig. 1).

In adults, intrinsic functional connectivity is thought to reflect the brain's functional architecture, which emerges as a result of task-elicited coactivation between brain regions (Fox and Raichle, 2007). Children's intrinsic functional connectivity patterns are likely constructed in a similar manner; however, the long-term molding hypothesis has proposed that these connectivity patterns are shaped over time as a result of both maturation and experience (Gabard-Durnam et al., 2016). For example, by following 4- to 18-year-olds over 2 years, prospective analyses indicated that task-elicited amygdala functional connectivity predicted resting-state functional connectivity 2 years later, but not concurrently (Gabard-Durnam et al., 2016). These findings suggest associations between task-based brain activation and intrinsic functional connectivity in both children and adults; however, such associations may differ across development.

Empirical data also support bidirectional influences between the brain and behavior. First, previous studies support the notion that behavioral changes shape task-based and intrinsic functional connectivity (e.g., Jolles et al., 2016; Clark et al., 2017; Rosenberg-Lee et al., 2018). For example, in 8- to 9-yearold children, 8 weeks of math tutoring strengthened iHFC to intraparietal sulcus (Jolles et al., 2016). Second, empirical studies have shown that intrinsic functional connectivity can predict gains in cognitive abilities later in development (e.g., Hoeft et al., 2011; Supekar et al., 2013; Evans et al., 2015). For example, Supekar et al. (2013) found that iHFC measured before math tutoring predicted performance improvements after tutoring during middle childhood.

Based on these studies, we explored whether there were bidirectional influences between source memory changes and intrinsic functional connectivity from the hippocampus to brain regions reported to support encoding contextual information (Geng et al., 2019). We used an accelerated longitudinal design to assess the following: (a) age-related changes in concurrent relations between source memory and iHFC; (b) predictive relations between early source memory changes and later iHFC; and (c) predictive relations between early iHFC and later source memory changes (Fig. 1).

We hypothesized that early source memory gains would predict later iHFC because the impact of experience is thought to build up over time (Gabard-Durnam et al., 2016). Accumulating experiences with everyday memory activities were expected to drive developmental changes in iHFC during childhood. Additionally, because of greater plasticity early in development (e.g., Tottenham and Sheridan, 2010), we expected that memory changes would more robustly predict later connectivity in the younger versus older cohort.

As brain connectivity has been suggested to shape later behavior (e.g., Evans et al., 2015), we hypothesized that iHFC would predict gains in source memory abilities. Additionally, because hippocampal function is more mature during middle versus early childhood (Geng et al., 2019), we hypothesized that connectivity at 6 years would more robustly predict future memory than connectivity at 4 years.

\section{Materials and Methods}

\section{Participants}

Children were participants in a large study investigating memory and brain development in early childhood that used an accelerated longitudinal design ( $N=200,100$ males) (Riggins et al., 2018; Geng et al., 2019). The first wave (W1) of the study included 4- to 8-year-old children. The 


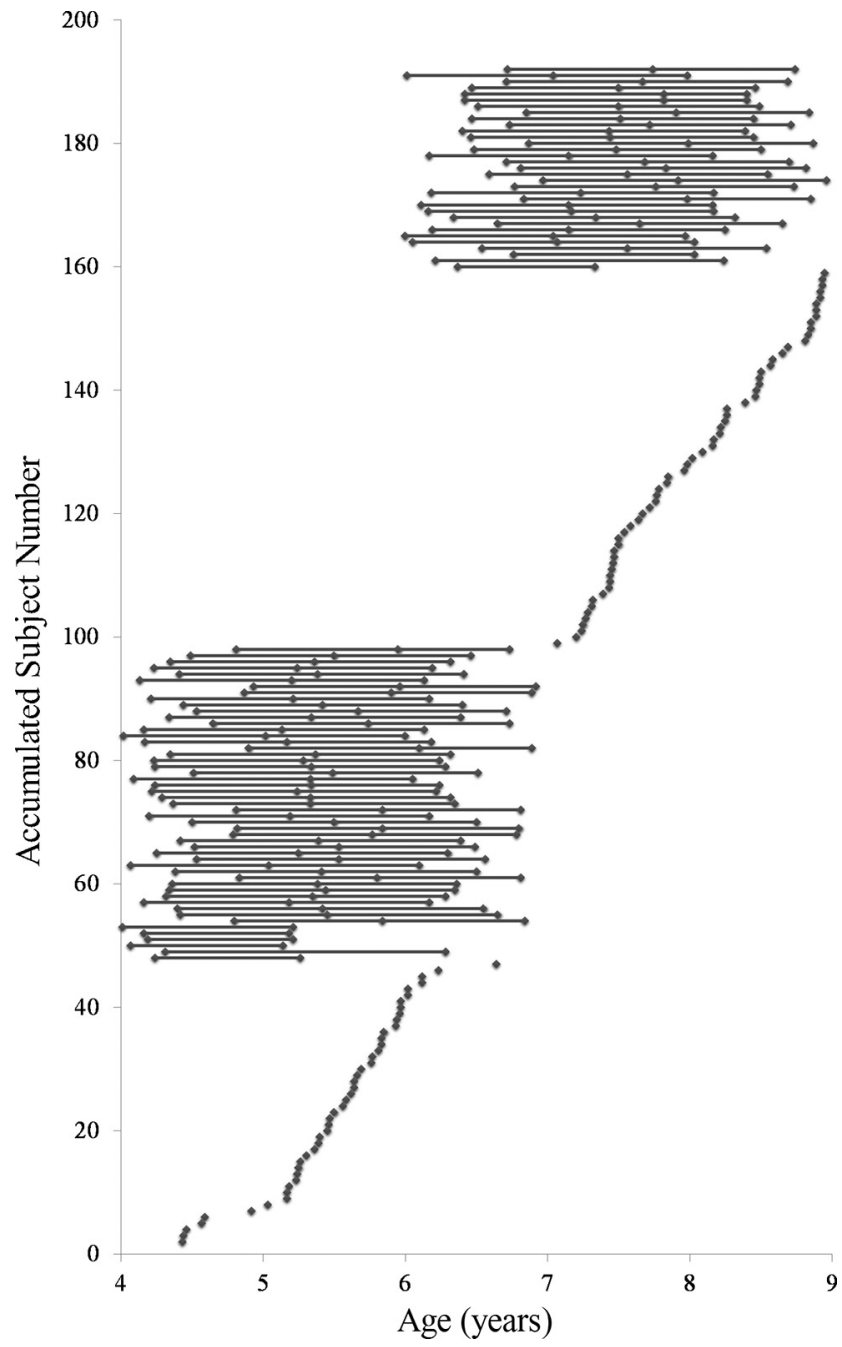

Figure 2. Number of waves children participated in the study and their age at each wave of participation.

4- and 6-year-old children were invited back for two subsequent waves of testing (W2 and W3; Fig. 2). In total, there were three waves, and each wave included young and old cohorts (young cohort: $\mathrm{W} 1=4$ years, $\mathrm{W} 2=5$ years, $\mathrm{W} 3=6$ years; old cohort: $\mathrm{W} 1=6$ years, $\mathrm{W} 2=7$ years, $\mathrm{W} 3=8$ years). Table 1 shows the number of children who provided 3, 2 , or 1 waves of data for final analyses in each cohort. The main reasons for loss of neuroimaging data were that the children moved too much, fell asleep during the scan, refused to enter the scanner, or the families failed to follow up.

We measured children's IQ at W1 by using the vocabulary and block design subtests from either the Wechsler Intelligence Scale for Children (Ed 4) (Wechsler, 2003) or the Wechsler Preschool and Primary Scale for Intelligence (Wechsler, 2012). All children included in analyses had average to above-average estimated IQ. No difference was found between the young and old cohorts in scaled scores on the vocabulary (young: mean $=11.07, \mathrm{SD}=2.96$; old: mean $=11.66, \mathrm{SD}=2.55 ; p=0.33$ ) or block design subtests (young: mean $=12.96, \mathrm{SD}=2.73$; old: mean $=13.03, \mathrm{SD}=3.00 ; p=0.92$ ). Parents reported all participants to be healthy without any neurodevelopmental disorders, neurologic conditions, or psychiatric conditions. Additionally, parents reported $88.5 \%$ children as righthanded, $6.8 \%$ as lefthanded, $3.7 \%$ as ambidextrous, and $1 \%$ as not able to be determined.

\section{Experimental design}

Encoding. During the first visit, children learned novel facts (e.g., "A group of rhinos is called a crash") from one of two different sources, a female adult ("Abby") and a male-voiced puppet ("Henry") via digital videos (Drummey and Newcombe, 2002; Riggins, 2014). Each source provided 6 facts for a total of 12 facts. Presentation of facts was blocked by source, where children first learned 6 facts from one source followed by 6 facts from the other source, and the order of blocks was randomized across participants. There were three lists of facts; each list consisted of unique facts that were similar across lists (e.g., "A group of kangaroos is called a mob" or "A group of goats is called a tribe"). These lists were randomly assigned across participants. Children were asked to pay attention to the facts as they would be tested on the facts the following week, but were not told that they would be tested on the source of the facts. Children were asked whether they knew the facts before the experiment. Known facts were excluded and were replaced with additional novel facts from the list of the same source (but this rarely occurred). Each source had 8 possible facts to account for the possibility that children would know 1 or 2 of the facts. If a child knew 3 or more facts from one source, the total number of facts the child was tested on was reduced (but this was rare, $n=4)$.

Retrieval. During the second visit, children were tested on their memory for the facts and sources from the first visit. Children were asked to answer 22 trivia questions and to tell the experimenter where they had learned the answers to those trivia questions. They were told that they had learned some of the questions the week before from either "Abby" or "Henry," some they might have learned outside the laboratory (e.g., from a teacher or parent), and some they may not know. The children learned 6 of the 22 facts presented from "Abby," 6 from "Henry," 5 were facts commonly known by children (e.g., "What color is the sky?"), and 5 were facts that children typically would not know (e.g., "What is the colored part of your eye called?"). Each list of 22 facts had two random presentation orders, and these orders were counterbalanced across participants. If children were unable to recall the source for a particular question, five multiple choice options were given: parents, teacher, girl in the video, puppet in the video, or just knew/guessed.

Source memory was calculated as the proportion of questions for which children accurately recalled or recognized both the fact and the source out of the total number of facts learned (see the formula below). This measure of source memory is thought to reflect the binding of facts and sources, which is an important aspect of episodic memory (Miller et al., 2013; Cooper and Ritchey, 2020).

\section{Source memory}

$$
=\frac{\text { Number of recognized or recalled sources out of remembered facts }}{\text { Total number of learned facts }}
$$

\section{Imaging data acquisition}

Participants were scanned in a Siemens 3.0 T scanner (MAGNETOM Trio Tim System, Siemens Medical Solutions) using a 32-channel coil. Children first completed the task-free scan, followed by a T1-weighted structural scan (T1, see Riggins et al., 2018). In the first wave of data collection, children also completed a memory encoding task if time permitted (for results, see Geng et al., 2019). During the task-free scan, children were not given any overt task but were instructed to lie as still as possible with their eyes open. The duration of the scan was $7 \mathrm{~min}$ and $6 \mathrm{~s}$. To minimize motion, Inscapes, a movie designed to reduce head motion during fMRI data collection, was played (Vanderwal et al., 2015). A total of 210 whole-brain volumes were collected using a T2 $*$-weighted gradient EPI sequence (TR $2 \mathrm{~s}$, TE $25 \mathrm{~ms}$, slice thickness $3.5 \mathrm{~mm}$, voxel size $3.0 \mathrm{~mm} \times 3.0 \mathrm{~mm} \times 3.5 \mathrm{~mm}$, voxel matrix $64 \times 64$, flip angle $70^{\circ}$, FOV $192 \mathrm{~mm}, 36$ slices). Structural images were acquired during a $4 \mathrm{~min} 26 \mathrm{~s}$ scan with a T1-weighted MPRAGE (TR $1.9 \mathrm{~s}$; TE $2.32 \mathrm{~ms}$; slice thickness $0.9 \mathrm{~mm}$ with no gap; voxel size $0.9 \times 0.9 \times 0.9 \mathrm{~mm}$; voxel matrix $256 \times 256 \mathrm{~mm}$; flip angle $9^{\circ}$; FOV $230 \times 230 \mathrm{~mm}$ ).

During the task-free scan, participant head motion was monitored in real-time. If a participant exhibited excessive head motion $(>2 \mathrm{~mm}$ in any direction) during the first half of any run, the scan was restarted and the participant was reminded to stay as still as possible. 
Table 1. Participants included in behavioral and neuroimaging data analyses

\begin{tabular}{|c|c|c|c|}
\hline$n$ of waves & Cohort & Behavioral ( $n$ of subjects) & Neuroimaging ( $n$ of subjects) \\
\hline \multirow[t]{2}{*}{ Subjects who had data for 3 waves } & Young cohort $(4,5$, and $6 \mathrm{yr})$ & 45 & 25 \\
\hline & Old cohort $(6,7$, and $8 \mathrm{yr})$ & 32 & 25 \\
\hline \multirow[t]{2}{*}{ Subjects who had data for 2 waves } & Young cohort $(4,5$, or 6 yr) & 6 & 14 \\
\hline & Old cohort $(6,7$, or 8 yr $)$ & 3 & 9 \\
\hline \multirow[t]{2}{*}{ Subjects who had data for 1 wave } & Young cohort $(4,5$, or 6 yr) & 47 & 39 \\
\hline & Old cohort $(6,7$, or $8 \mathrm{yr})$ & 61 & 58 \\
\hline Grand total & & 194 & 170 \\
\hline
\end{tabular}

Table 2. Sample size and motion parameters (mean, SD) of all age groups for each analysis ${ }^{a}$

\begin{tabular}{|c|c|c|c|c|c|}
\hline Analyses & Group & Sample size & Mean FD & Absolute movement in FD & Data length (min) \\
\hline \multirow[t]{5}{*}{ Age-related change in concurrent relations between behavior and brain } & $4 \mathrm{yr}$ & 38 & $0.21(0.10)$ & $0.24(0.19)$ & $6.56(0.58)$ \\
\hline & $5 \mathrm{yr}$ & 59 & $0.23(0.12)$ & $0.24(0.13)$ & $6.40(0.56)$ \\
\hline & $6 \mathrm{yr}$ & 77 & $0.20(0.10)$ & $0.22(0.14)$ & $6.59(0.48)$ \\
\hline & $7 \mathrm{yr}$ & 58 & $0.20(0.09)$ & $0.23(0.12)$ & $6.54(0.52)$ \\
\hline & $8 \mathrm{yr}$ & 61 & $0.19(0.10)$ & $0.20(0.11)$ & $6.64(0.41)$ \\
\hline \multirow[t]{2}{*}{ Behavioral change predicting brain } & Young cohort & 38 & $0.18(0.09)$ & $0.18(0.10)$ & $6.71(0.39)$ \\
\hline & Old cohort & 36 & $0.17(0.08)$ & $0.18(0.08)$ & $6.71(0.30)$ \\
\hline \multirow[t]{2}{*}{ Brain predicting behavioral change } & Young cohort & 33 & $0.22(0.10)$ & $0.26(0.20)$ & $6.53(0.59)$ \\
\hline & Old cohort & 31 & $0.21(0.11)$ & $0.24(0.14)$ & $6.50(0.54)$ \\
\hline
\end{tabular}

${ }^{a}$ There were no significant differences between groups in all motion parameters.

Imaging data preprocessing

In the analyses, all 210 collected resting-state fMRI images were included, as the first four volumes were discarded before data collection because of the instability of the initial MR signal and participant adaptation. Preprocessing included the following steps. First, slice time correction, head motion correction, and smoothing were performed using DPABI 1.3 (Yan et al., 2016). An independent component analysis was then run on smoothed data using MELODIC, an FSL toolbox, to remove artifact-related components (Geng et al., 2019). After removing all artifact-related components, brain extraction, normalization, and filtering were conducted. Following the procedure suggested by Tillman et al. (2018), brain extraction on T1-weighted image was conducted separately in six toolboxes: the Advanced Normalization Tools, AFNI, FSL, BSE, ROBEX, and SPM8 to ensure high-quality data. The voxels extracted by at least four toolboxes were included in the brain mask. Advanced Normalization Tools was used to perform coregistration and normalization. Statistical analyses were conducted in AFNI (Cox, 1996). Temporal bandpass filtering $(0.01-0.1 \mathrm{~Hz})$ and spatial smoothing with a $5 \mathrm{~mm}$ FWHM Gaussian kernel were performed in AFNI on normalized data.

Individual seed regions (bilateral anterior and posterior hippocampus) were derived from the structural scan using Freesurfer 5.1 (https:// surfer.nmr.mgh.harvard.edu) (Fischl, 2012) and edited using Automatic Segmentation Adapter Tool (www.nitrc.org/projects/segadapter) (Wang et al., 2011). The hippocampus was divided into anterior and posterior segments using manual identification of standard anatomic landmarks. The uncal apex served as the border between anterior and posterior hippocampus (Duvernoy, 2005; Weiss et al., 2005). Raters were blind to participant age and sex. Reliability for identification of these landmarks indicated $94.60 \%$ agreement within 1 slice and $99.99 \%$ agreement within 2 slices. Intraclass correlation coefficients were high and ranged from 0.897 to 0.985 . When there was disagreement between raters on the correct slice location, the more experienced rater's slice was used.

Task-free functional connectivity analyses were conducted in AFNI. First, to minimize the effect of head motion, volumes with framewise displacement $(\mathrm{FD}) \geq 0.5 \mathrm{~mm}$ were scrubbed in addition to 1 volume before and 1 volume after the offending volume. All children included in the final statistical analyses had data $\geq 4.87 \mathrm{~min}$ in length and mean FD from 0.05 to $0.50(0.20 \pm 0.10$, mean $\pm \mathrm{SD})$. Mean FD, absolute movement, and data length for each age group are reported in Table 2. There was a marginally significant relation between age and mean FD for the analyses testing the age-related changes in concurrent relations using the fixed effects model $(\beta=-0.011, \mathrm{SE}=0.006, t=-1.773, p=0.077)$. For the two prediction analyses, independent $t$ tests indicated that there were no differences in any motion parameters between young and old cohorts $(p>0.70)$.

Correlations between the time series of the individual seed regions (bilateral anterior and posterior hippocampus) and those of the whole brain were calculated to generate individual resting state functional connectivity maps. Subsequently, Fisher's $r$-to- $z$ transformation was used to convert $r$ maps into $z$ maps to obtain normally distributed values of the connectivity maps. The $z$ values were extracted from 6 ROIs: inferior/superior parietal lobule (IPL/SPL), inferior occipital gyrus (IOG), left inferior temporal gyrus (ITG), left inferior frontal gyrus (IFG), fusiform gyrus, and orbital frontal gyrus (OFG) (Fig. 3). These brain regions were defined according to previous research, which indicated that 4- to 8-year-old children showed greater activation in these regions during encoding for items (i.e., pictures of animal and objects) subsequently remembered with correct sources than the ones with incorrect sources (i.e., pictures of cartoon characters) (for details, see Geng et al., 2019). The activation differences suggest that these six brain regions are critical for encoding contextual information. Therefore, these regions were chosen as ROIs in the current study.

\section{Statistical analyses}

Linear mixed models were used given their capability to handle unbalanced and incomplete longitudinal data. Specifically, a series of fixed effects models were run in SPSS 20.0 to test the associations between age, source memory, and iHFC. First, age-related changes in source memory were assessed. Then, relations between age, source memory, and iHFC to each ROI were analyzed to assess the changes in concurrent relations. Next, the models were used to examine whether earlier changes in source memory predicted later iHFC with each ROI and whether earlier functional connectivity with each ROI predicted later changes in source memory.

As suggested in the Introduction, it takes time for behavioral and brain changes to mold each other. Therefore, we analyzed the relations of memory changes between W1 and W2 to functional connectivity at W3 (Fig. 1). In addition, we calculated the relations of functional connectivity at $\mathrm{W} 1$ to memory changes between $\mathrm{W} 1$ and $\mathrm{W} 3$ (Fig. 1). Another practical reason for why the memory change intervals were different between the two prediction analyses was that there was no significant change in source memory between W2 and W3 in the old cohort. [Another question of interest would be how changes in source memory are related to changes in iHFC; however, in the current sample, there was too much data loss because of motion to examine changes in iHFC within the two cohorts. Therefore, single time point data are used for functional connectivity analyses.] 


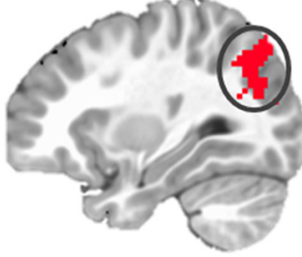

$\mathrm{x}=-30$

Left IPL/SPL

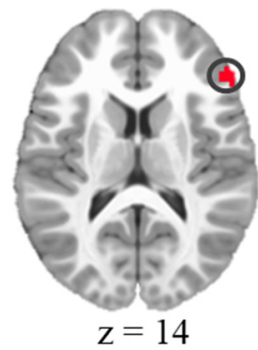

Left IFG

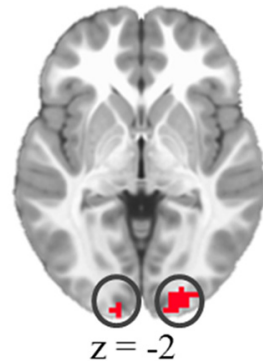

IOG

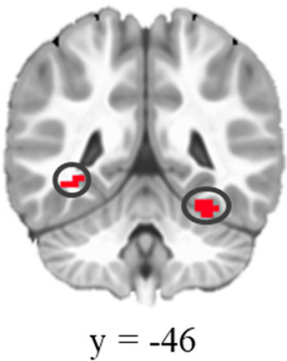

Fusiform

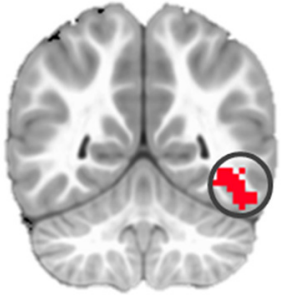

$\mathrm{y}=-53$

Left ITG

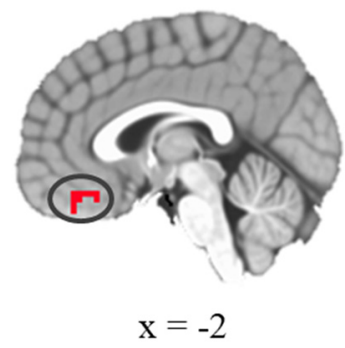

OFG

Figure 3. ROls used in hippocampal functional connectivity analyses. Adapted from Geng et al. (2019).
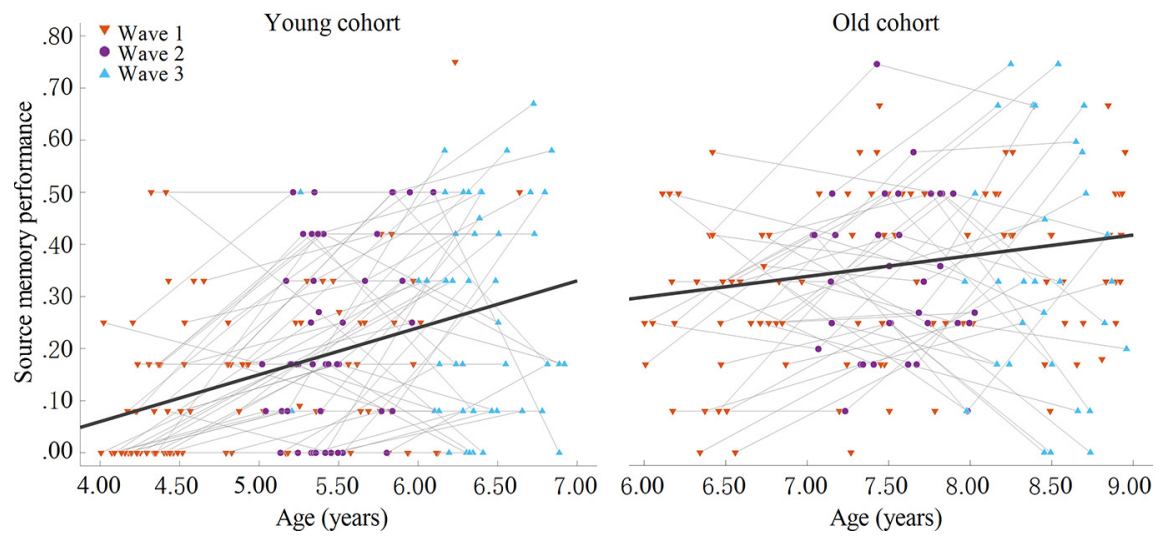

Figure 4. Relations between age and memory performance for each cohort.

Continuous covariates (age, mean FD, source memory change, and iHFC) in these models were standardized across the full sample. Akaike's Information Criterion was used to compare models of main effects to models that included both main and interactive effects. Models with the lowest Akaike's Information Criterion value were selected as the best fitting model. Given the moderate sample size (see Table 1) and the limited number of ROI, corrections for multiple comparisons were not applied. If interactions involving cohort (young vs old) were observed, the relation between source memory measures and iHFC was estimated again for each cohort by restandardizing the covariates for each group separately and re-estimating model parameters.

For all linear mixed models involving iHFC as the dependent measure, bilateral hippocampal subregion (anterior vs posterior) was included as a within-subjects factor. Since differences between hippocampal subregions have been reported in a previous study (Blankenship et al., 2017), results related to subregion differences are reported briefly.

\section{Results}

Behavioral results

There were 194 children who provided data for behavioral analyses (Table 1). To predict source memory performance, fixed effects models, including age, cohort (young vs old), and an age $\times$ cohort interaction had better fit than the model that only included main effects. Age was positively related to source memory performance ( $\beta=0.051$, $\left.\mathrm{SE}=0.023, t_{(353)}=2.281, p=0.023\right)$, and there was a significant interaction between age and cohort $(\beta=-0.075, \mathrm{SE}=0.031$, $\left.t_{(353)}=-2.423, p=0.016\right)$. When this interaction was probed, results showed that age-related changes in source memory were greater in the young versus old cohort ( $p<0.001$ vs $p=0.028$; Fig. 4 ). No differences were observed between the 6year-old children from the young cohort who were tested at W3 and the 6-year-old children from the old cohort who were tested at W1 $(p>0.90)$. Additionally, we tested whether there were differences in source memory between age groups in each cohort. All the comparisons between age groups reached significance with the exception of the difference between 7 and 8 years $(p=0.24)$.

\section{Age-related changes in concurrent} relations between source memory and functional connectivity

Fixed effects of the linear mixed models included subregion, age, and source memory as independent variables and iHFC as the dependent variable. Mean FD was also included in the model because of the marginally significant relation between age and mean FD as reported above. The main effects models had better fit than the models with age $\times$ source memory interaction. There were significant main effects of age in predicting the connectivity between hippocampus and left IPL/SPL, IOG, left ITG, left IFG, fusiform gyrus, and OFG. Specifically, as age increased, iHFC increased as well (Fig. 5). The main effect of source memory was not significant for the connectivity between hippocampus and any ROI.

Additionally, there were differences in the connectivity between anterior versus posterior hippocampus and fusiform, left ITG, IOG, OFG, and left IPL/SPL (Table 3). In all regions except OFG, connectivity was greater for posterior compared with anterior hippocampus. In IFG, there was a trending difference in hippocampal functional connectivity (anterior $<$ posterior; Table 3 ).

\section{Predictive relations between source memory changes from $\mathrm{W} 1$ to $\mathrm{W} 2$ and functional connectivity at W3}

Fixed effects of the linear mixed models included subregion (anterior vs posterior), cohort (young vs old), and source memory change (defined as the differences in source memory between W1 and W2). Mean FD was not included in the models because there was no difference in any of the motion parameters (mean FD, absolute movement, and data length) between young and old cohorts $(p>0.60)$. The models containing the cohort $\times$ source memory change interaction resulted in better model fit 

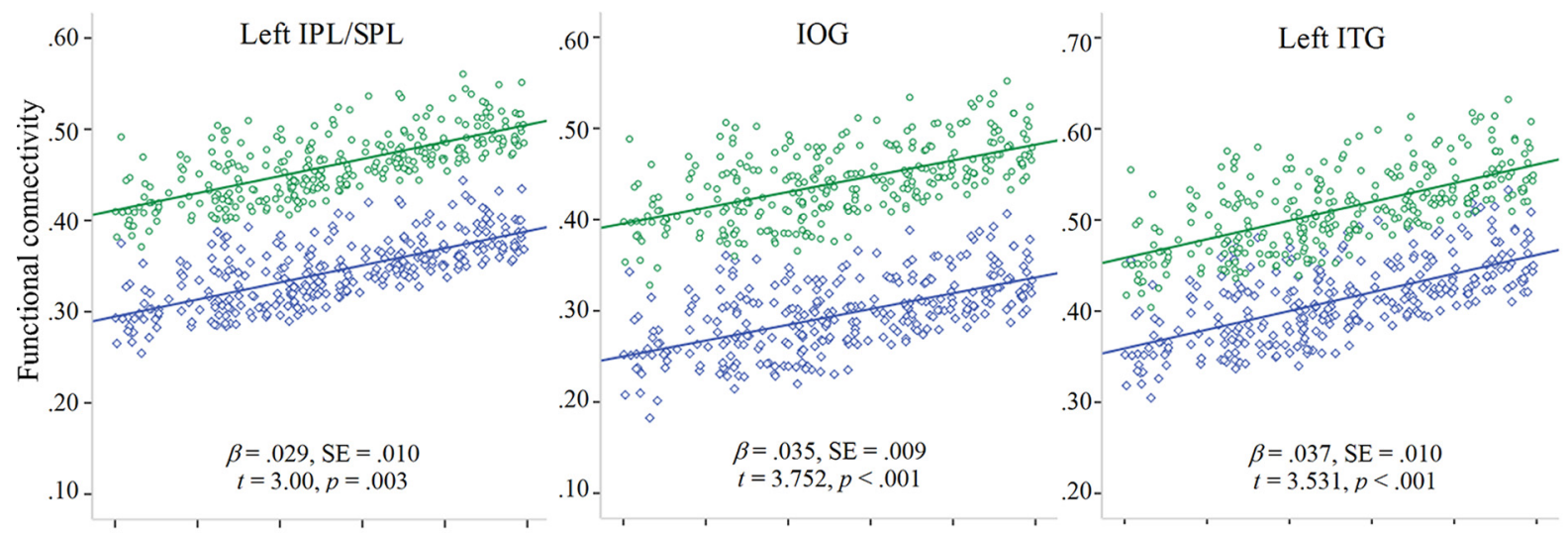

$\diamond$ Anterior
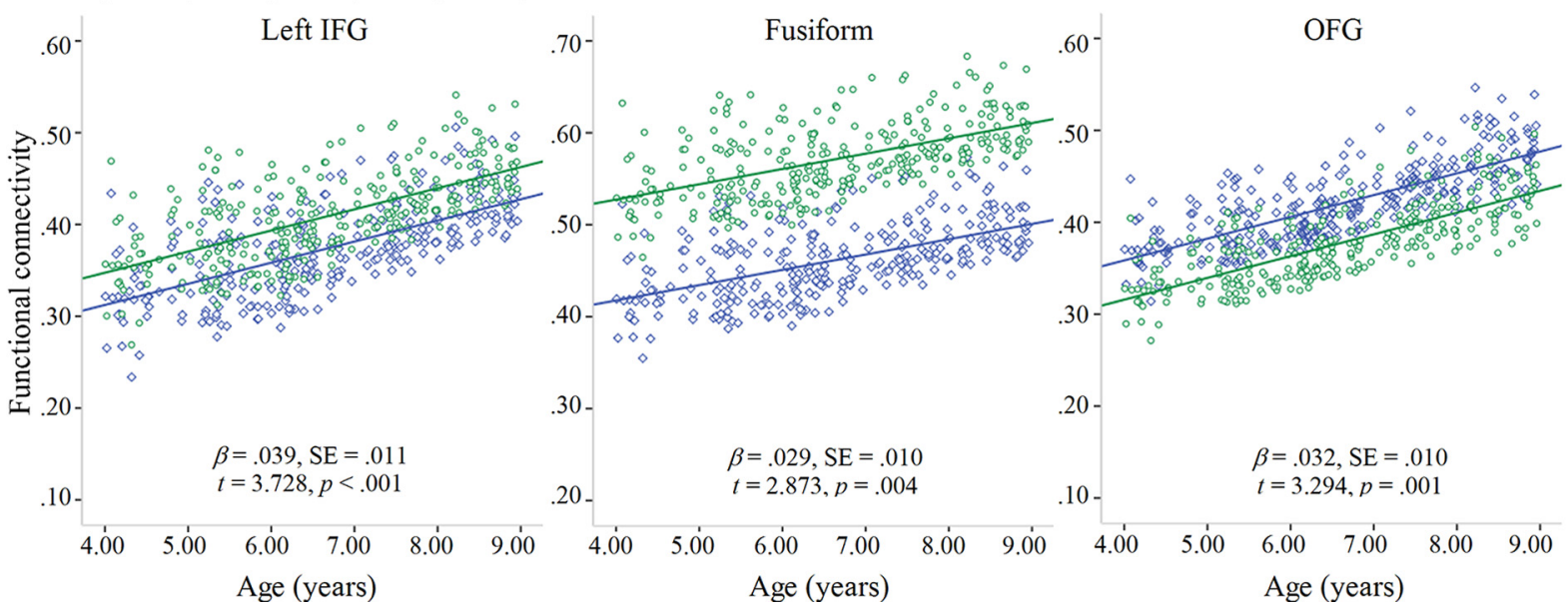

o Posterior

Figure 5. Significant associations between age and iHFC with each ROI. Green represents fit line for anterior hippocampus. Blue represents fit line for posterior hippocampus.

Table 3. Differences in iHFC between anterior and posterior hippocampus

\begin{tabular}{|c|c|c|c|c|c|c|c|}
\hline \multirow[b]{2}{*}{ Subregion (anterior vs posterior) } & & \multicolumn{6}{|c|}{ Hippocampal functional connectivity (dependent variable) } \\
\hline & & Left IPS/SPL & IOG & Left ITG & Left IFG & Fusiform & OFG \\
\hline \multirow{3}{*}{ Age-related change in concurrent relations between behavior and brain } & $\beta$ & -0.117 & -0.146 & -0.099 & -0.035 & -0.110 & 0.043 \\
\hline & $t$ & -6.684 & -8.882 & -5.363 & -1.870 & -6.013 & 2.463 \\
\hline & $p$ & $<0.001^{*}$ & $<0.001^{*}$ & $<0.001^{*}$ & 0.062 & $<0.001^{*}$ & $0.014^{*}$ \\
\hline \multirow[t]{2}{*}{ Behavioral change predicting brain } & $\beta$ & -0.093 & -0.148 & -0.084 & -0.022 & -0.128 & 0.047 \\
\hline & $p$ & $0.012^{*}$ & $<0.001^{*}$ & $0.042^{*}$ & 0.477 & $0.001^{*}$ & 0.195 \\
\hline
\end{tabular}

${ }^{*} p<.05$.

for left IFG and OFG. In contrast, the main effects models had better fit for the other four ROIs. Source memory changes between $\mathrm{W} 1$ and $\mathrm{W} 2$ were positively related to W3 hippocampal functional connectivity with left IPL/SPL, IOG, left ITG, and fusiform gyrus (Table 4; Fig. 6).

There were significant interactions between cohort and source memory change predicting intrinsic functional connectivity from hippocampus to left IFG and OFG (Table 4). To probe these interactions, the fixed effects models were used to assess each cohort separately. In the young cohort, source memory changes were significantly related to iHFC with left IFG and OFG $\left(\beta=0.109, \mathrm{SE}=0.025, t_{(73)}=4.341, p<0.001\right.$; $\left.\beta=0.068, \mathrm{SE}=0.022, t_{(73)}=3.117, p=0.003\right)$. However, in the old cohort, these associations were not apparent ( $p$ values $>0.20$ ). Therefore, only in the young cohort did behavioral improvements in source memory shape later intrinsic functional connectivity between hippocampus and these cortical regions (Fig. 7). Moreover, we reran the analyses above with source memory at W1 and W2 included in the models to test the relations between source memory changes and later hippocampal functional connectivity. All results stayed the same, except that the main effect of source memory changes in left IPL/SPL and IOG became nonsignificant $(p=0.18 ; p=0.11)$.

Additionally, posterior hippocampus showed greater connectivity to fusiform, left ITG, IOG, and left IPL/SPL than anterior hippocampus. The difference in OFG and left IFG did not reach significance (Table 3).

\section{Predictive relations between functional connectivity at $\mathrm{W} 1$ and source memory changes from $\mathrm{W} 1$ to $\mathrm{W} 3$}

Fixed effects models included iHFC at W1 and cohort (young vs old) as independent variables and source memory change from 
Table 4. Relations between source memory changes from W1 to W2 and iHFC at $\mathrm{W}^{\mathrm{a}}$

\begin{tabular}{|c|c|c|c|c|c|c|c|}
\hline \multirow[b]{2}{*}{ Model (independent variable) } & & \multicolumn{6}{|c|}{ Hippocampal functional connectivity (dependent variable) } \\
\hline & & Left IPS/SPL & IOG & Left ITG & Left IFG & Fusiform & OFG \\
\hline \multirow[t]{3}{*}{ Cohort } & $\beta$ & 0.007 & -0.019 & -0.011 & -0.063 & -0.013 & -0.054 \\
\hline & $t$ & 0.186 & -0.536 & -0.251 & -1.570 & -0.329 & -1.478 \\
\hline & $p$ & 0.852 & 0.593 & 0.802 & 0.119 & 0.743 & 0.142 \\
\hline \multirow[t]{2}{*}{ Memory change } & $\beta$ & 0.061 & 0.080 & 0.076 & 0.020 & 0.047 & -0.370 \\
\hline & $p$ & $0.002^{*}$ & $<0.001^{*}$ & $<0.001^{*}$ & 0.477 & $0.016^{*}$ & 0.151 \\
\hline \multirow[t]{4}{*}{ Cohort $\times$ Memory change } & $\beta$ & - & - & - & 0.096 & - & 0.110 \\
\hline & SE & - & - & - & 0.040 & - & 0.037 \\
\hline & $t$ & - & - & - & 2.398 & - & 2.989 \\
\hline & $p$ & - & - & - & $0.018^{*}$ & - & $0.003^{*}$ \\
\hline
\end{tabular}

${ }^{a}$ Subregion was also included in the models as an independent measure. Statistical details for this variable are presented in Table 3. —, Interaction effect was not included in the model. ${ }^{*} p<.05$.
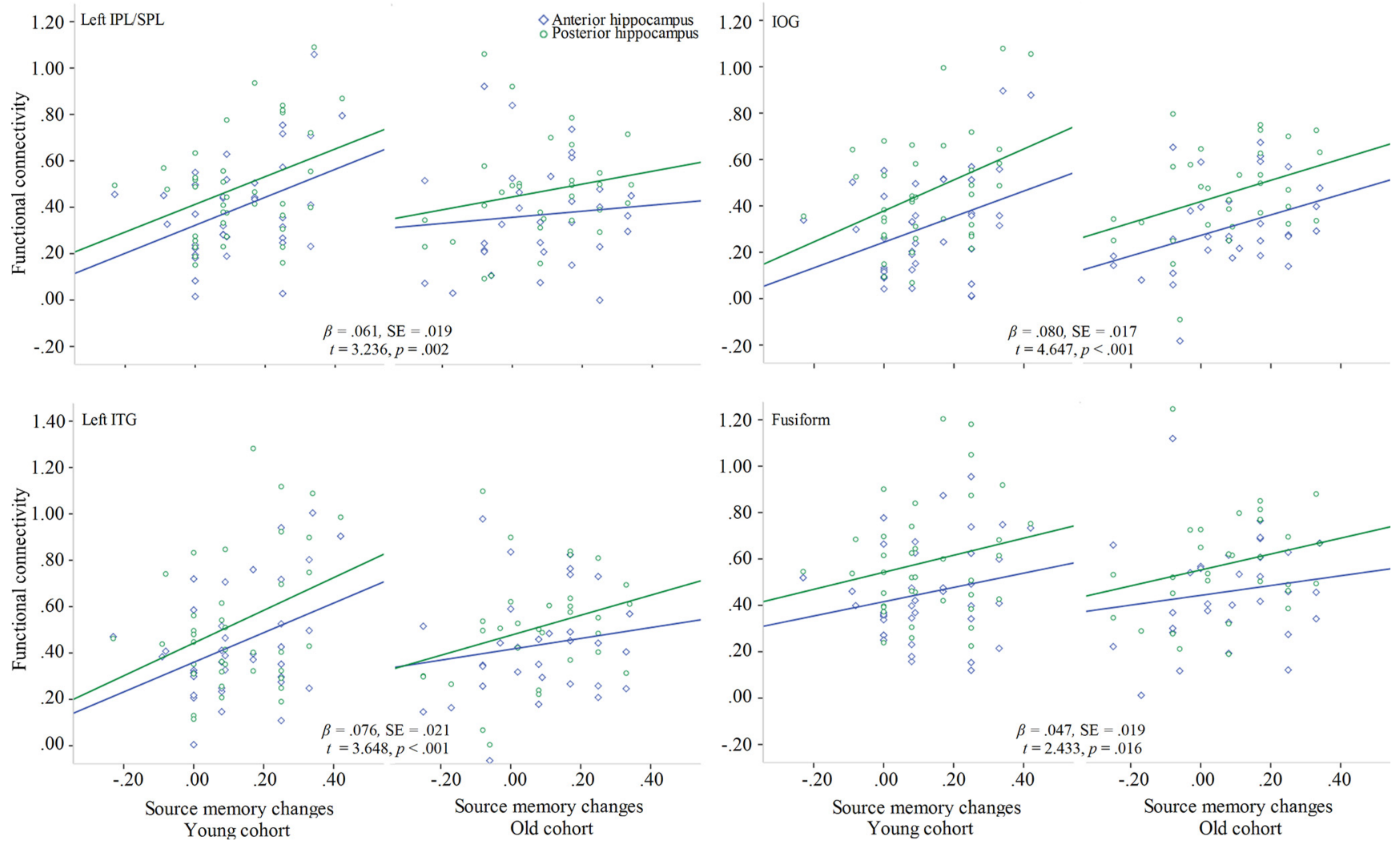

Figure 6. Plots represent the relations between source memory changes and functional connectivity for Rols showing a main effect of source memory change.

W1 to W3 as the dependent variable. Mean FD was not included in the model as it was not significantly different between cohorts $(p>0.70)$. For left ITG and fusiform, the interaction models had better fit. For the other ROIs, the main effects models had better fit but did not generate any significant finding.

The interactions between cohort and iHFC in left ITG and fusiform gyrus significantly predicted source memory change (Table 5). To probe the interactions, fixed effects models were used to test the relations in each cohort separately. In the older cohort, iHFC with left ITG and fusiform gyrus was related to source memory change $\left(\beta=-0.090, \mathrm{SE}=0.024, t_{(60)}=3.76\right.$, $\left.p<0.001 ; \beta=0.086, \mathrm{SE}=0.029, t_{(60)}=2.998, p=0.004\right)$. However, this association was not observed in the young cohort $(p>0.80)$. Therefore, only in the old cohort did functional connectivity between hippocampus and left ITG and fusiform gyrus predict later improvements in source memory (Fig. 7).

\section{Discussion}

This study used an accelerated longitudinal design to investigate the predictive relations between source memory changes and iHFC across childhood. First, we found age-related increases in source memory performance, supporting previous studies highlighting age-related differences in source memory in this age group (Drummey and Newcombe, 2002; Riggins, 2014). Second, results indicated that iHFC within the memory network increased across childhood, which is consistent with previous cross-sectional studies showing similar associations between age and hippocampal 
Source memory predicts functional connectivity
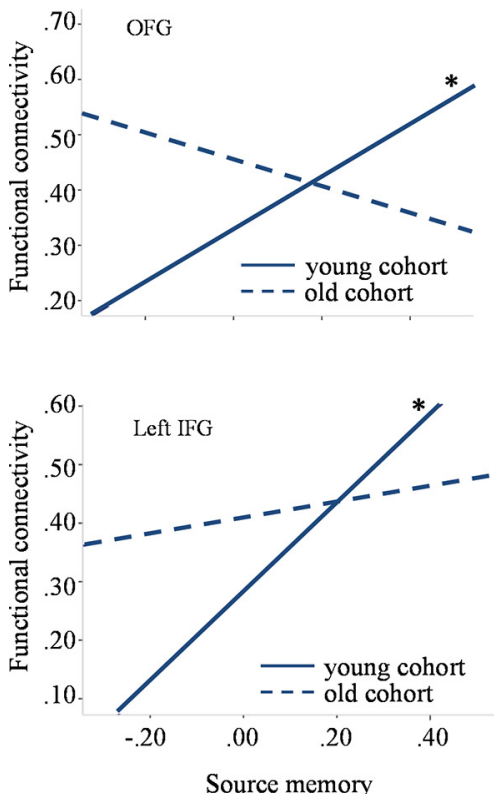

Functional connectivity predicts source memory
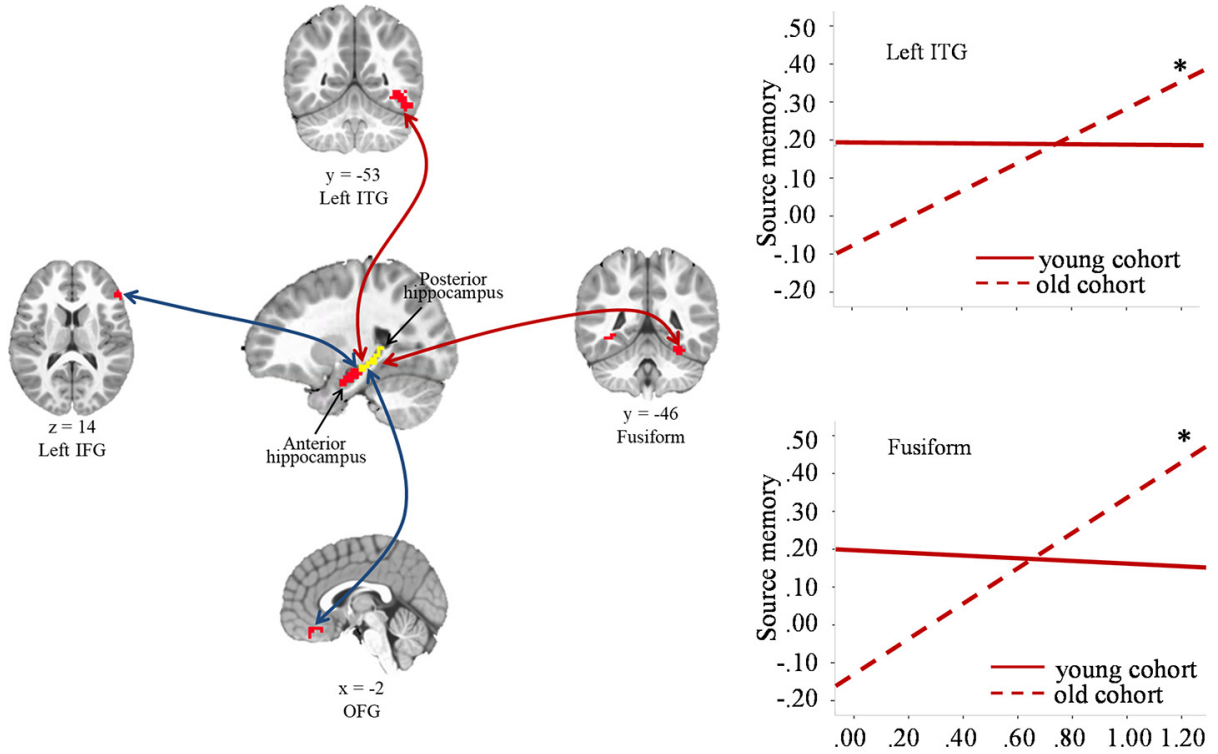

Functional connectivity

Figure 7. Plots represent associations between source memory changes and iHFC for each cohort from analyses showing significant interactive effects. $* p<0.01$.

Table 5. Relations between iHFC at W1 and source memory change from W1 to W3 ${ }^{\mathrm{a}}$

\begin{tabular}{|c|c|c|c|c|c|c|c|}
\hline \multirow[b]{2}{*}{ Model (independent variable) } & & \multicolumn{6}{|c|}{ Source memory change (dependent variable) } \\
\hline & & Left IPS/SPL & IOG & Left ITG & Left IFG & Fusiform & OFG \\
\hline \multirow[t]{4}{*}{ Cohort } & $\beta$ & 0.094 & 0.099 & 0.096 & 0.095 & 0.090 & 0.097 \\
\hline & SE & 0.039 & 0.040 & 0.039 & 0.040 & 0.038 & 0.040 \\
\hline & $t$ & 2.385 & 2.475 & 2.474 & 2.412 & 2.348 & 2.429 \\
\hline & $p$ & $0.019^{*}$ & $0.015^{*}$ & $0.015^{*}$ & $0.017^{*}$ & $0.020^{*}$ & $0.017^{*}$ \\
\hline \multirow[t]{4}{*}{ iHFC } & $\beta$ & 0.039 & 0.011 & 0.086 & 0.034 & 0.090 & 0.026 \\
\hline & SE & 0.020 & 0.027 & 0.028 & 0.020 & 0.024 & 0.030 \\
\hline & $t$ & 1.973 & 0.399 & 3.024 & 1.665 & 3.723 & 0.849 \\
\hline & $p$ & 0.051 & 0.691 & $0.003^{*}$ & 0.099 & $<0.001^{*}$ & 0.398 \\
\hline \multirow[t]{4}{*}{ Cohort $\times$ iHFC } & $\beta$ & - & - & -0.086 & - & -0.096 & - \\
\hline & SE & - & - & 0.039 & - & 0.039 & - \\
\hline & $t$ & - & - & -2.194 & - & -2.439 & - \\
\hline & $p$ & - & - & $0.030^{*}$ & - & $0.016^{*}$ & - \\
\hline
\end{tabular}

a_, Interaction effect was not included in the model.

${ }^{*} p<.05$.

functional connectivity (Blankenship et al., 2017; Geng et al., 2019). Finally, results indicated that changes in memory performance predicted later functional connectivity and early functional connectivity predicted later source memory changes.

\section{Source memory changes predicted later hippocampal functional connectivity}

Source memory changes early in life predicted later intrinsic connectivity between the hippocampus and cortical regions in the absence of a task, suggesting that behavior shaped later functional connectivity. Specifically, across all children, changes in memory predicted intrinsic connectivity from hippocampus to fusiform, left ITG, IOG, and left IPL/SPL 1 year later. Additionally, within the younger cohort, early memory changes also predicted hippocampal functional connectivity with left IFG and OFG. However, in analyses assessing aged-related changes in concurrent relations, memory ability was not related to iHFC. These findings support the idea that life experience shapes the brain across development, a notion that has received support from other findings as well (Fox and Raichle, 2007; Gabard-Durnam et al., 2016). For instance, task-elicited functional connectivity has been shown to predict resting-state functional connectivity 2 years later but not concurrently (Gabard-Durnam et al., 2016). Additionally, as suggested by the interactive specialization framework, we interpreted the findings to show that changes in memory early in life may drive functional integration within the hippocampal memory network and that this process takes time to unfold (Johnson, 2011; Poppenk et al., 2013). In other words, early memory gains may reflect gradual refinement of the hippocampal memory network over time, which ultimately leads to enhanced iHFC even during the absence of a specific task. This is consistent with previous research in other cognitive domains showing that arithmetic training induced changes in hippocampal-parietal connectivity in middle childhood (Rosenberg-Lee et al., 2018). 


\section{Hippocampal functional connectivity predicted later source memory changes}

Connectivity from hippocampus to fusiform and ITG predicted memory changes 2 years later. However, this was only true for the older cohort, such that iHFC with fusiform and left ITF at 6 years predicted memory changes 2 years later. These findings support the claim that activity in the brain can also shape behavior, and suggest that functional integration within the hippocampal memory network can predict future developmental gains in memory ability in children as young as 6 years of age. This finding is consistent with previous studies suggesting that earlier iHFC can predict intervention-induced behavioral changes in typical and atypical developmental populations (Hoeft et al., 2011; Supekar et al., 2013). Therefore, our study suggests that, early in life, the interaction between brain and behavioral development is not unidirectional, but instead a reciprocal process: behavioral changes shape future brain activity, and brain activity shapes future behavioral changes.

\section{Timing effects in the interaction between behavioral and brain development}

Our results suggest that timing matters in terms of the interactions between brain and behavioral development. Specifically, although earlier memory changes predicted later hippocampal connectivity with certain cortical regions in both young and old cohorts, only within the younger cohort were early memory changes related to the functional connectivity from hippocampus to left IFG and OFG. In contrast, early functional connectivity with fusiform and left ITG significantly predicted later memory changes in the older cohort only. We propose two possible explanations of these timing differences. First, these findings may result from known differences in the magnitude of agerelated changes in the development of source memory during childhood. Consistent with previous behavioral studies suggesting accelerated rates of change in source memory before the sixth year of life (Riggins, 2014), the current study indicates that memory changes for the 4-to-6-year old cohort were more substantial than for the 6-to-8-year old cohort. Greater memory changes may drive greater changes in the brain. This may help explain why earlier memory changes in the younger cohort predicted connectivity between the hippocampus and IFG and OFG.

Alternatively, the timing difference may result from known differences in developmental changes in the hippocampus during childhood. Studies in humans and animals consistently indicate that the hippocampal memory network experiences significant development around the sixth year of life (Serres, 2001; Lavenex and Lavenex, 2013). Therefore, 4-6 years may be a sensitive period for hippocampal development, characterized by greater plasticity (Tottenham and Sheridan, 2010). This claim is consistent with the argument that shaping functional architecture may be particularly pronounced during developmental periods when neural systems are most plastic and amenable to environmental inputs (Bick and Nelson, 2017). After the sensitive period ends, the more mature hippocampal memory network may begin driving developmental changes in memory abilities. This may explain why iHFC predicted behavioral changes in the older cohort only. Therefore, we speculate that only after the brain reaches a certain threshold of maturation can its function reliably and consistently predict behavior.

The timing effects may also depend on the specific brain regions exhibiting functional connectivity with the hippocampus. Early memory changes from 4 to 5 years predicted functional connectivity between hippocampus and prefrontal regions (IFG and OFG) at 6 years. However, in the older cohort, functional connectivity at age 8 years was not predicted by behavior changes from 6 to 7 years. In contrast, only in the older cohort did iHFC predict memory changes 2 years later, and this was specific to the fusiform and ITG. These findings may be because of the different developmental trajectories of these neural regions. Previous research has shown that brain development proceeds in a hierarchical sequence from sensory to association cortices to regions important for cognitive functions (Gogtay et al., 2004; Shaw et al., 2008). IFG and OFG are regions associated with higher-order cognition that mature later than sensory or association regions. Thus, the findings of the current study are consistent with the notion that prefrontal regions, such as IFG and OFG, are less mature, and therefore more plastic, in the younger versus older cohort. This is also consistent with previous studies indicating that increased hippocampal functional coupling with the PFC is related to the development of memory abilities (Qin et al., 2014). The present findings extend this work and suggest that 4-6 years may be a sensitive period for the functional integration between hippocampus and frontal regions. In contrast, fusiform and ITG are related to visual processing and mature earlier than prefrontal regions. Such early maturation might underlie the ability of fusiform and ITG to predict behavioral changes.

\section{Strengths and limitations}

The current study has several strengths. By using an accelerated longitudinal design, we traced developmental changes in source memory, iHFC, and relations between them. This design also allowed us to test timing differences by comparing two cohorts of children. By focusing on a proposed sensitive period for the development of source memory, we were able to examine how the hippocampal memory network is refined within this important developmental window and how it relates to improvements in memory.

This study also has limitations. Because of the limited sample size, we were unable to examine how changes in functional connectivity over time relate to memory. Also, because of the sample size, we elected not to conduct whole-brain analyses and instead relied on ROIs derived from a task-based study involving children from the same longitudinal study. Thus, findings with additional brain regions, not included in the current set of ROIs, may also exist. Additionally, the ROIs were defined in a task using only pictorial material. In contrast, source memory in this study was measured in a task involving both pictorial and verbal material. It is unknown whether different brain regions would be activated to encode contextual information tapping visual versus verbal modalities. Finally, although a proportion of children in the sample provided task-based fMRI data to define ROIs, we did not have enough task-based MRI data to assess developmental relations between the task-based hippocampal functional connectivity and the connectivity measured without a demanding task imposed.

In conclusion, our study focused on the mechanisms related to developmental changes in source memory and the underlying hippocampal network between 4 and 8 years of age. Results indicate that behavioral experience and brain changes are interactive processes, such that experience shapes changes in the brain and the brain shapes changes in behavior. Additionally, our findings support and extend previous studies of brain development by showing that timing matters in terms of behavioral changes molding brain connectivity and such timing differences also depend on which brain regions are involved. Together, these findings add critical insight into the development of source 
memory in early childhood and contribute to the growing body of literature documenting the interactive and intricate relations between cognitive processes and their neurologic bases in the developing brain.

\section{References}

Bauer PJ (2007) Event memory. In: Handbook of child psychology. New York: Wiley.

Bick J, Nelson CA (2017) Early experience and brain development. Wiley Interdiscip Rev Cogn Sci 8:e1387.

Blankenship SL, Redcay E, Dougherty LR, Riggins T (2017) Development of hippocampal functional connectivity during childhood. Hum Brain Mapp 38:182-201.

Clark R, Wendel C, Voss MW (2017) Physical activity and cognitive training: impact on hippocampal structure and function. In: The hippocampus from cells to systems. Cham: Springer.

Cooper RA, Ritchey M (2020) Progression from feature-specific brain activity to hippocampal binding during episodic encoding. J Neurosci 40:17011709.

Cox RW (1996) AFNI: software for analysis and visualization of functional magnetic resonance neuroimages. Comput Biomed Res 29:162-173.

Drummey AB, Newcombe NS (2002) Developmental changes in source memory. Dev Sci 5:502-513.

Duvernoy HM (2005) The human hippocampus: functional anatomy, vascularization and serial sections with MRI. Berlin: Springer.

Evans TM, Kochalka J, Ngoon TJ, Wu SS, Qin S, Battista C, Menon V (2015) Brain structural integrity and intrinsic functional connectivity forecast 6 year longitudinal growth in children's numerical abilities. J Neurosci 35:11743-11750.

Fischl B (2012) FreeSurfer. Neuroimage 62:774-781.

Fox MD, Raichle ME (2007) Spontaneous fluctuations in brain activity observed with functional magnetic resonance imaging. Nat Rev Neurosci 8:700-711.

Gabard-Durnam LJ, Gee DG, Goff B, Flannery J, Telzer E, Humphreys KL, Lumian DS, Fareri DS, Caldera C, Tottenham N (2016) Stimulus-elicited connectivity influences resting-state connectivity years later in human development: a prospective study. J Neurosci 36:4771-4784.

Geng F, Redcay E, Riggins T (2019) The influence of age and performance on hippocampal function and the encoding of contextual information in early childhood. Neuroimage 195:433-443.

Ghetti S, Bunge SA (2012) Neural changes underlying the development of episodic memory during middle childhood. Dev Cogn Neurosci 2:381-395.

Gogtay N, Giedd JN, Lusk L, Hayashi KM, Greenstein D, Vaituzis AC, Nugent TF, Herman DH, Clasen LS, Toga AW, Rapoport JL, Thompson PM (2004) Dynamic mapping of human cortical development during childhood through early adulthood. Proc Natl Acad Sci USA 101:81748179.

Hoeft F, McCandliss BD, Black JM, Gantman A, Zakerani N, Hulme C, Lyytinen H, Whitfield-Gabrieli S, Glover GH, Reiss AL, Gabrieli JDE (2011) Neural systems predicting long-term outcome in dyslexia. Proc Natl Acad Sci USA 108:361-366.

Johnson MH (2011) Interactive specialization: a domain-general framework for human functional brain development? Dev Cogn Neurosci 1:7-21.

Jolles D, Supekar K, Richardson J, Tenison C, Ashkenazi S, Rosenberg-Lee M, Fuchs L, Menon V (2016) Reconfiguration of parietal circuits with cognitive tutoring in elementary school children. Cortex 83:231-245.

Lavenex P, Lavenex BP (2013) Building hippocampal circuits to learn and remember: insights into the development of human memory. Behav Brain Res 254:8-21.
Miller JF, Neufang M, Solway A, Brandt A, Trippel M, Mader I, Hefft S, Merkow M, Polyn SM, Jacobs J, Kahana MJ, Schulze-Bonhage A (2013) Neural activity in human hippocampal formation reveals the spatial context of retrieved memories. Science 342:1111-1114.

Poppenk J, Evensmoen HR, Moscovitch M, Nadel L (2013) Long-axis specialization of the human hippocampus. Trends Cogn Sci 17:230-240.

Qin S, Cho S, Chen T, Rosenberg-Lee M, Geary DC, Menon V (2014) Hippocampal-neocortical functional reorganization underlies children's cognitive development. Nat Neurosci 17:1263-1269.

Riggins T (2014) Longitudinal investigation of source memory reveals different developmental trajectories for item memory and binding. Dev Psychol 50:449-459.

Riggins T, Geng F, Botdorf M, Canada K, Cox L, Hancock GR (2018) Protracted hippocampal development is associated with age-related improvements in memory during early childhood. Neuroimage 174:127137.

Riggins T, Canada KL, Botdorf M (2020) Empirical evidence supporting neural contributions to episodic memory development in early childhood: implications for childhood amnesia. Child Dev Perspect 14:41-48.

Rosenberg-Lee M, Iuculano T, Bae SR, Richardson J, Qin S, Jolles D, Menon V (2018) Short-term cognitive training recapitulates hippocampal functional changes associated with one year of longitudinal skill development. Trends Neurosci Educ 10:19-29.

Sastre M, Wendelken C, Lee JK, Bunge SA, Ghetti S (2016) Age- and performance-related differences in hippocampal contributions to episodic retrieval. Dev Cogn Neurosci 19:42-50.

Serres L (2001) Morphological changes of the human hippocampal formation from midgestation to early childhood. In: Handbook of developmental cognitive neuroscience (Nelson CA, Luciana M, eds), pp 45-58. Cambridge, MA: Massachusetts Institute of Technology.

Shaw P, Kabani NJ, Lerch JP, Eckstrand K, Lenroot R, Gogtay N, Greenstein D, Clasen L, Evans A, Rapoport JL, Giedd JN, Wise SP (2008) Neurodevelopmental trajectories of the human cerebral cortex. J Neurosci 28:3586-3594.

Supekar K, Swigart AG, Tenison C, Jolles DD, Rosenberg-Lee M, Fuchs L, Menon V (2013) Neural predictors of individual differences in response to math tutoring in primary-grade school children. Proc Natl Acad Sci USA 110:8230-8235.

Tang L, Shafer AT, Ofen N (2018) Prefrontal cortex contributions to the development of memory formation. Cereb Cortex 28:3295-3308.

Tottenham N, Sheridan M (2010) A review of adversity, the amygdala and the hippocampus: a consideration of developmental timing. Front Hum Neurosci 3:68

Vanderwal T, Kelly C, Eilbott J, Mayes LC, Castellanos FX (2015) Inscapes: a movie paradigm to improve compliance in functional magnetic resonance imaging. Neuroimage 122:222-232.

Wang H, Das SR, Suh JW, Altinay M, Pluta J, Craige C, Avants B, Yushkevich PA, Alzheimer's Disease Neuroimaging Initiative (2011) A learning-based wrapper method to correct systematic errors in automatic image segmentation: consistently improved performance in hippocampus, cortex and brain segmentation. Neuroimage 55:968-985.

Wechsler D (2003) Wechsler Intelligence Scale for Children-Fourth Edition (WISC-IV). San Antonio, TX: The Psychological Corporation.

Wechsler D (2012) Wechsler Preschool and Primary Scale of IntelligenceFourth Edition. San Antonio, TX: The Psychological Corporation.

Weiss AP, DeWitt I, Goff D, Ditman T, Heckers S (2005) Anterior and posterior hippocampal volumes in schizophrenia. Schizophr Res 73:103-112.

Yan CG, Wang XD, Zuo XN, Zang YF (2016) DPABI: data processing and analysis for (resting-state) brain imaging. Neuroinformatics 14:339-351. 